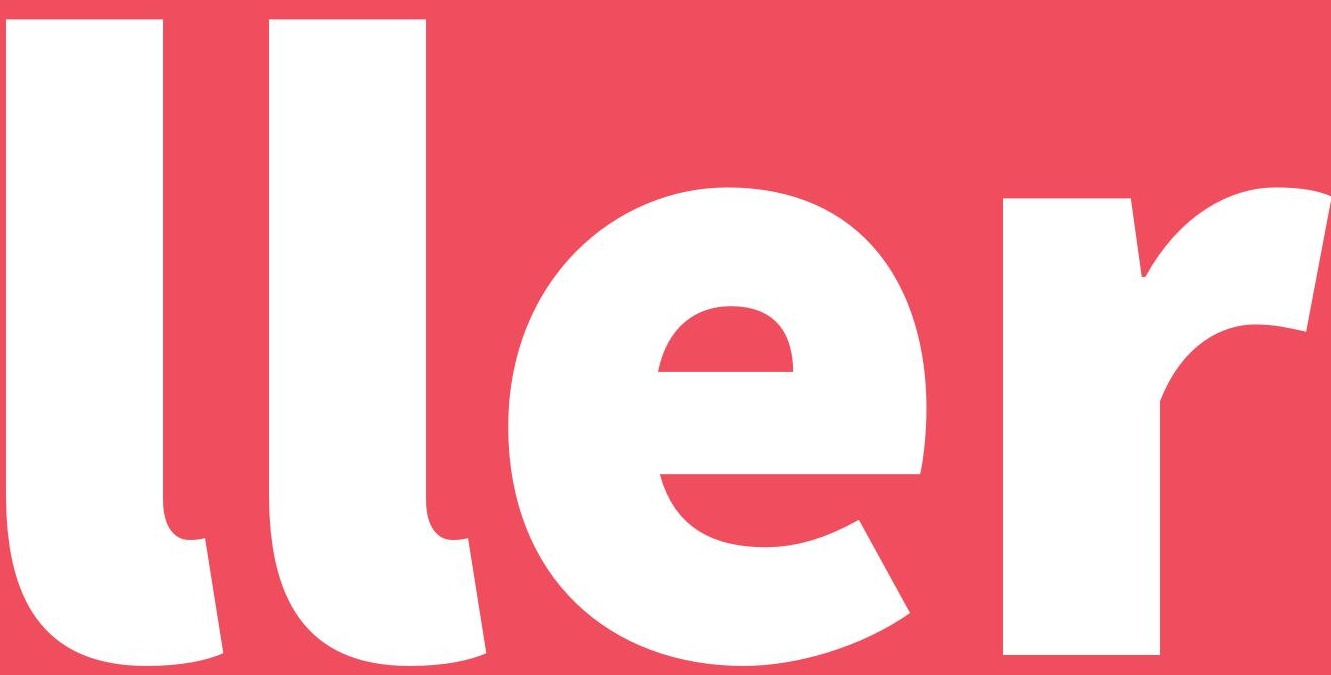

\title{
El Proyecto
}

de Diseño

como motor

de innovación

en empresas

regionales

del Risaralda:

Casos BUSSCAR

de Colombia y

HANKO Leather Crafts

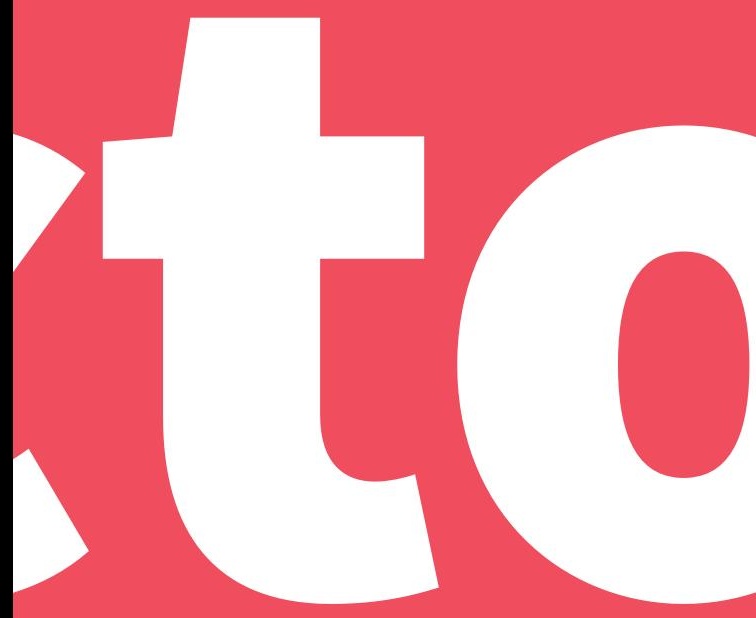

Gustavo Adolfo

Peña Marín ${ }^{1}$

Carlos Andrés

Quintero Diaztagle

\section{Resumen}

Esta experiencia educativa evidencia las diversas etapas de un proyecto académico de diseño tecnológico hecho con estudiantes
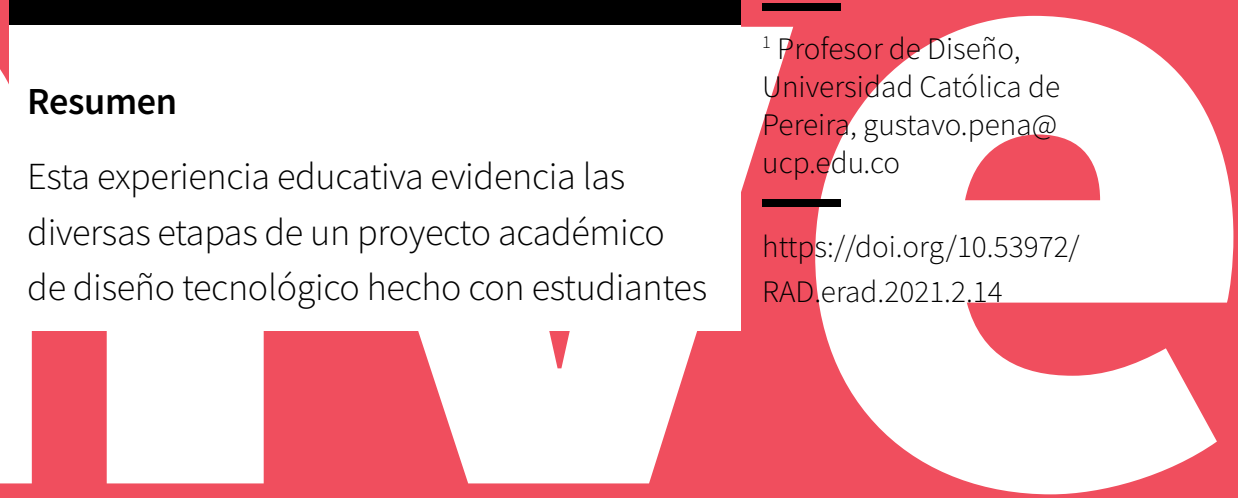
del Programa de Diseño Industrial de la Universidad Católica de Pereira (UCP) y dos empresas industriales en el departamento de Risaralda durante el segundo semestre de 2020, como una oportunidad para reflexionar las didácticas virtuales requeridas para encontrar soluciones innovadoras en estos entornos. Las actividades desarrolladas tuvieron un enfoque prospectivo que permitieron la innovación de sus productos de cara a la nueva realidad suscitada por la pandemia del Covid-19, lo que requirió métodos de diseño con carácter tecnológico productivo y la implementación de herramientas digitales para el modelado de prototipos. Ello incluyó realidades extendidas en la virtualidad a través de trabajo remoto. El primer caso industrial abordado fue con la empresa BUSSCAR de Colombia para generar nuevas estrategias de transporte terrestre de personas en la nueva realidad para dos de sus vehículos. El segundo caso fue en la empresa HANKO Leather Crafts, donde se requirió el desarrollo de accesorios vestibles en cuero con características tecno-sensoriales para generar nuevas interacciones humanas tipo cyborg. Estos ejercicios proyectuales durante la pandemia arrojan diversas conclusiones Palabras clave con el apoyo de la industria. 


\section{Introducción}

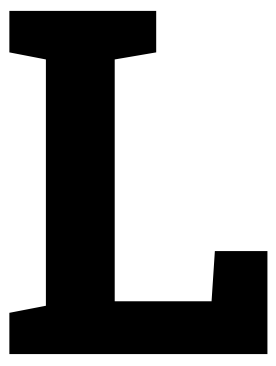

Las universidades al igual que las empresas industriales de la región del eje cafetero colombiano han sostenido su esfuerzo constante para sobrevivir al impacto causado por el suceso mundial de la pandemia del Covid-19. Una de las alternativas generales que han encontrado son el diseño de nuevas estrategias para mantener en funcionamiento sus actividades regulares en servicios educativos para el caso de las universidades y soportar la demanda y fabricación de productos transformados industrialmente como las empresas de manufactura respectivamente. Las dificultades para la enseñanza y el aprendizaje durante la pandemia basados en métodos tradicionales que implican la presencialidad revelaron la necesidad de asumir definitivamente los modelos alternativos para la educación diseñados para la distancia y la virtualidad (Blu Radio, 2021). Estos modelos alternativos implican el uso de tecnologías de mediación para facilitar la interacción, especialmente en los procesos educativos de carácter proyectual como el caso de los programas de diseño con necesidades especiales para dinamizar sus didácticas y métodos de transmisión del conocimiento.

Algunos expertos han explorado y fomentado estas prácticas hace algunos años a 
nivel nacional. Tal es el caso de los colegios virtuales pioneros y algunas universidades con experiencia al respecto. Dichas instituciones han asumido el reto de superar la presencialidad y la formación a distancia típica para adentrarse en la formación virtual y el e-learning como posibilidad de formación integral (Cardona Ossa, 2006).

De igual modo, para la industria regional y quizás la nacional, ha sido ineludible la implementación de tecnologías de comunicación remota para facilitar la interacción de los colaboradores, tanto para la gestión de las áreas administrativas como el intercambio de datos e información, incluso de operación remota de tecnología (hardware y software) para mantener el aparato productivo en funcionamiento. En ese sentido, los procesos creativos adelantados en las áreas empresariales encargadas de la innovación de los productos y servicios que ellas ofrecen han tenido que aproximarse a modelos de trabajo propios de la co-creación y el co-diseño. Estos se basan en estrategias virtuales en plataformas que permiten no solo la comunicación bidireccional de los participantes, sino que integran diversas herramientas (apps, plug-ins, software remoto, etc.) para comunicar y representar los contenidos multimediales a nivel visual, sonoro y táctil. Factor que favorece la integración colectiva de los proyectos que se adelantan, incluso con licenciamiento abierto o de código libre para el acceso total de los participantes a estas sesiones de retroalimentación interactiva.

De ese modo, el Programa de Diseño Industrial de la Universidad Católica de Pereira ha apostado por integrarse con empresas industriales de la región para desarrollar alternativas conceptuales desde el diseño. Bajo tal iniciativa ha abordado ejercicios académicos que favorecen escenarios de oportunidad para resolver algunas necesidades de estas empresas en relación con sus productos y servicios. Gracias a ello ha atendido los desafíos que demanda la nueva realidad, observados desde la perspectiva creativa de los 
jóvenes estudiantes, quienes asumen en estos retos el liderazgo con espíritu innovador, con propuestas incrementales y en algunos casos radicales (Christensen, 2003).

Si bien, los estudiantes asumen una actitud conceptualmente propositiva sin descartar los avances tecnológicos más recientes que emergen en las búsquedas de tecnologías que pueden soportar la funcionalidad de sus propuestas de diseño, se evidencia que "la innovación incremental lleva menos tiempo e implica menos riesgo que la innovación radical" (Harvard Business Essentials, 2009, p.13). Por lo tanto, la viabilidad de las innovaciones para este ejercicio académico se consolida aprovechando la vigilancia tecnológica y las capacidades de los empresarios. Estos últimos, al abrir sus puertas comparten el know-how necesario con los estudiantes para impactar los mercados que atienden.

Figura $\mathbf{1}$ La tercera dimensión del modelo de innovación disruptiva

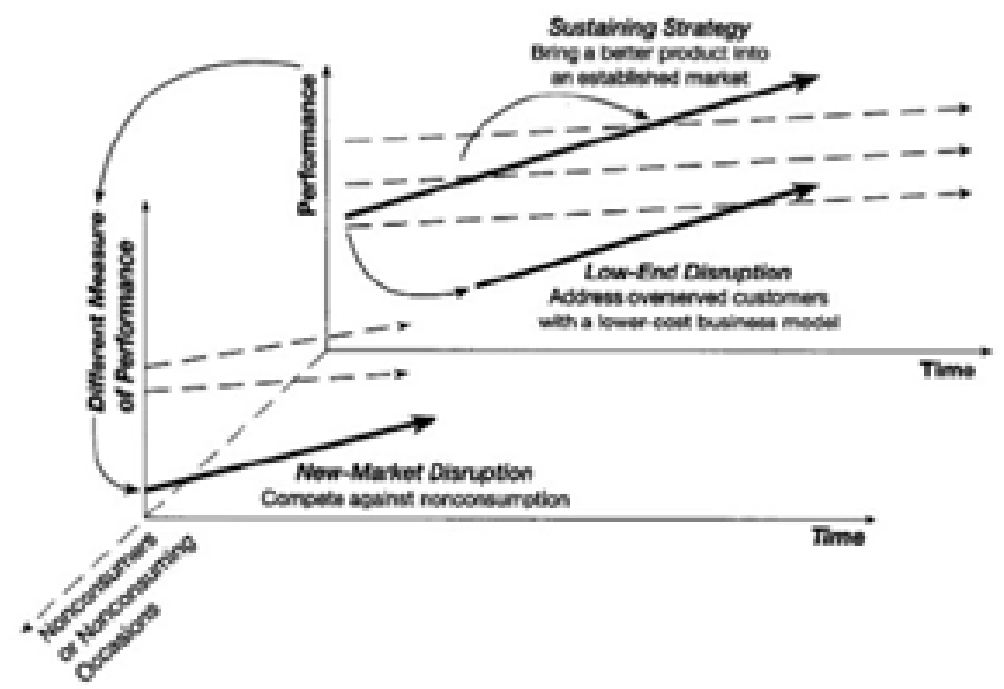

Fuente: Christensen, 2003. 
Desde esa lógica, se propende que los estudiantes exploren las posibilidades de intervenir en ambas empresas algunos de los productos con mayor potencial y necesidad de ser innovados. La idea es que esto se haga en razón al contexto presente y futuro que vive la humanidad con relación a los asuntos de bioseguridad y de interacciones naturales como experiencias diseñadas a través de gestos, expresiones y movimientos para descubrir el mundo mirando a su alrededor y manipulando cosas físicas (Valli, 2008). Factor que permitiría dinámicas saludables, confiables y fluidas al procurar encuentros humanizados entre los usuarios de estos productos.

\section{Metodologías, métodos e instrumentos de apoyo a los proyectos}

La propuesta epistemológica implementada por los docentes para el Taller de Proyectos se abordó desde múltiples elementos del Aprendizaje Basado en Problemas (ABP), lo que favoreció un ejercicio didáctico de tipo activo y de carácter significativo con 14 estudiantes de sexto semestre del Programa de Diseño Industrial. Las metodologías implementadas desde el Taller de Proyectos seis (tecnológico) se ajustaron a partir de algunos autores relevantes que sugieren estructuras flexibles y adaptativas como ruta para navegar las diferentes etapas de resolución de las problemáticas propuestas desde cada industria en particular. Inicialmente se sugiere la estructura general de la metodología Design Thinking en español como plataforma de trabajo desde sus cinco fases principales: Empatizar, definir, idear, prototipary testear. 
Figura 2 Metodología Design Thinking en español.

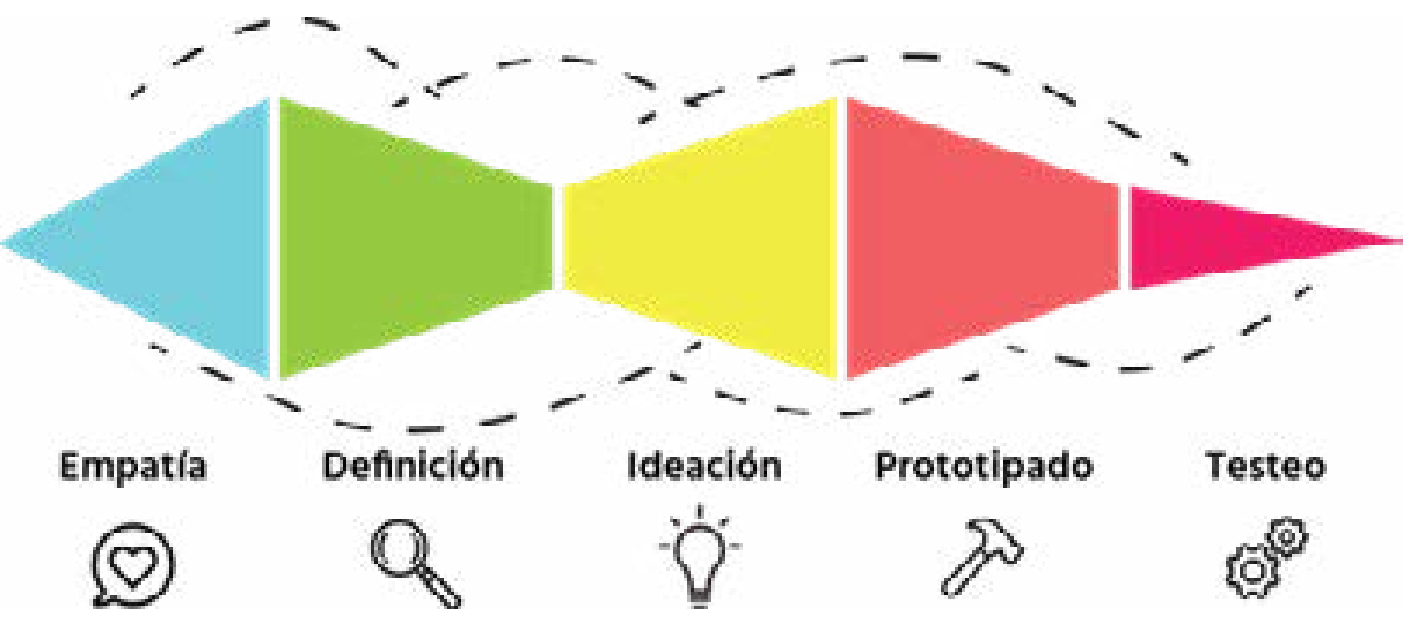

Fuente: designthinking.es (basado en IDEO)

La construcción del perfil de los potenciales usuarios de estas respuestas de diseño se definió a partir del método Persona (Kumar, 2013) al implementar encuestas con grupos de personas por medio virtuales como formularios en Google forms, Surveymonkey, entre otros instrumentos de uso libre. Por otro lado, se analizaron las posibles características tecnológicas con los entrevistados a través del método Kano Analysis (Hanington y Martin, 2019) para consolidar las preferencias técnicas y tecnológicas mejor adaptables a los perfiles de usuarios identificados.

De manera adicional, se implementaron algunos instrumentos de la Metodología TRIZ (Altshuller, 1997) como oportunidad para la resolución de problemas de inventiva. Esto se hizo tanto para aspectos relacionados con la ingeniería de los dispositivos sugeridos desde la vigilancia tecnológica hecha en diferentes portales y bases de datos (OMPI, Google Patents, entre otros) como instrumento de contrastación para verificar los criterios físico mecánicos y funcionales 
de los dispositivos que acompañan conceptualmente las alternativas de diseño propuestas. A continuación, se describen las particularidades de las dos experiencias proyectuales abordadas por los estudiantes de Taller de Proyectos seis (tecnológico) en alianza con estos actores de la realidad industrial, aprovechando las características de los entornos virtuales para favorecer el aprendizaje y potenciar la productividad.

\section{Experiencia 1: Busscar de Colombia}

La primera experiencia educativa se hizo con el acompañamiento de BUSSCAR de Colombia, empresa Pereirana dedicada al diseño y fabricación de vehículos de transporte masivo de personas con cobertura de mercados en toda Latinoamérica. La empresa requería que los estudiantes del Taller de Proyectos analizaran los espacio y las dinámicas de interacción dentro de sus buses para plantear una propuesta conceptual innovadora, que tenga como base los diversos aspectos de diseño al interior de estos ambientes respecto a la nueva realidad de la pandemia y el futuro del transporte terrestre.

El proyecto se tituló: Diseño e innovación para los buses de Busscar (Modelos: Urbanuss Pluss - Transporte masivo articulado/ biarticulado y Busstar Double Decker (DD S1) - Transporte intermunicipal doble piso).

De la mano del Director de Diseño de la compañía, se buscó involucrar a los estudiantes en una situación de diseño tecnológico conceptual. De tal manera que los estudiantes se expusieran a la mirada crítica de la empresa demandante de las innovaciones constantes al servicio de las personas. Es decir, el trabajo de los estudiantes era diseñar no solo la infraestructura necesaria para un viaje en bus, sino una experiencia de habitar los espacios en movimiento, incluso en situaciones que involucran interacciones humanas de manera satisfactoria, confortable, digna, segura y salubre para todos. 
El docente de Taller de Proyectos y el jefe de diseño de Busscar acordaron una serie de objetivos que cumpliesen tanto con las necesidades pedagógicas y profesionales de los estudiantes como las necesidades de la empresa. El resultado, fueron tres objetivos que se podrían expresar de la siguiente manera: En primer lugar, innovar conceptualmente el ambiente interior de los buses, sus accesorios y dinámicas de función-uso; segundo, generar alternativas de diseño a través de la investigación-creación; tercero, diseñar y optimizar virtualmente el espacio dentro de los buses seleccionados por Busscar. En adición a los objetivos anteriormente descritos, se esbozaron una serie de requerimientos iniciales que surgen a partir del contexto y condiciones de la empresa y de la realidad social. Estos se podrían expresar de la siguiente manera: (|1) Considerar en los diseños la situación actual de contingencia y la nueva realidad con relación al Covid-19; (2) el interior de los buses tendrá un concepto de diseño funcional sin discrepar con el estilo de Busscar; (3) respetar espacios, zonas y puntos para ventilación-extracción de aire, iluminación, salidas de emergencia, circulación, etc., como elementos técnicos inamovibles que las normas contemplan; (4) ubicar adecuadamente los diferentes servicios que se ofrecen dentro del bus en todos los planos y dimensiones, desde aspectos ergonómicos, normativos y técnicos a favor de la experiencia del usuario; (5) enfocar el trabajo de investigación directamente en el espacio del bus, más que en la indagación de campo con usuarios; (6) tener en cuenta proporciones correctas, se solicita la inclusión de monigotes (físicos y digitales) para escalar con la proporción real del espacio analizado; (7) se deben desarrollar elementos para compartir el transporte con el fin de favorecer el confort del usuario en trayectos cortos y largos, aprovechar las panorámicas, espacios entre sillas y configuraciones especiales; (8) mantener los costos bajos implementando solo los elementos necesarios, tal como se desarrollan actualmente los buses. A partir de dichos lineamientos, Busscar compartió datos e información 
general sobre medidas y otros asuntos de la empresa. Por ejemplo, como el tipo de tecnología implementada actualmente en los buses; asimismo, respondió a las preguntas de los estudiantes desde el día del lanzamiento del ejercicio y durante el proyecto. Algunas imágenes elaboradas por los estudiantes muestran los resultados logrados con la implementación de herramientas de visualización virtual para facilitar la comunicación de los proyectos y la sustentación remota durante la entrega definitiva con el equipo de diseñadores de la empresa.

Figura 3 Concepto Interior Bus Articulado-Materiales
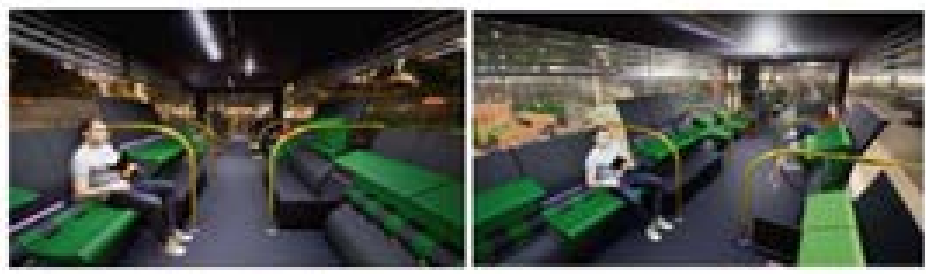

\section{Matrautrs}

Bioplast

Acero inoxidable (tuberia 27)

(3) Vidrio templado plano
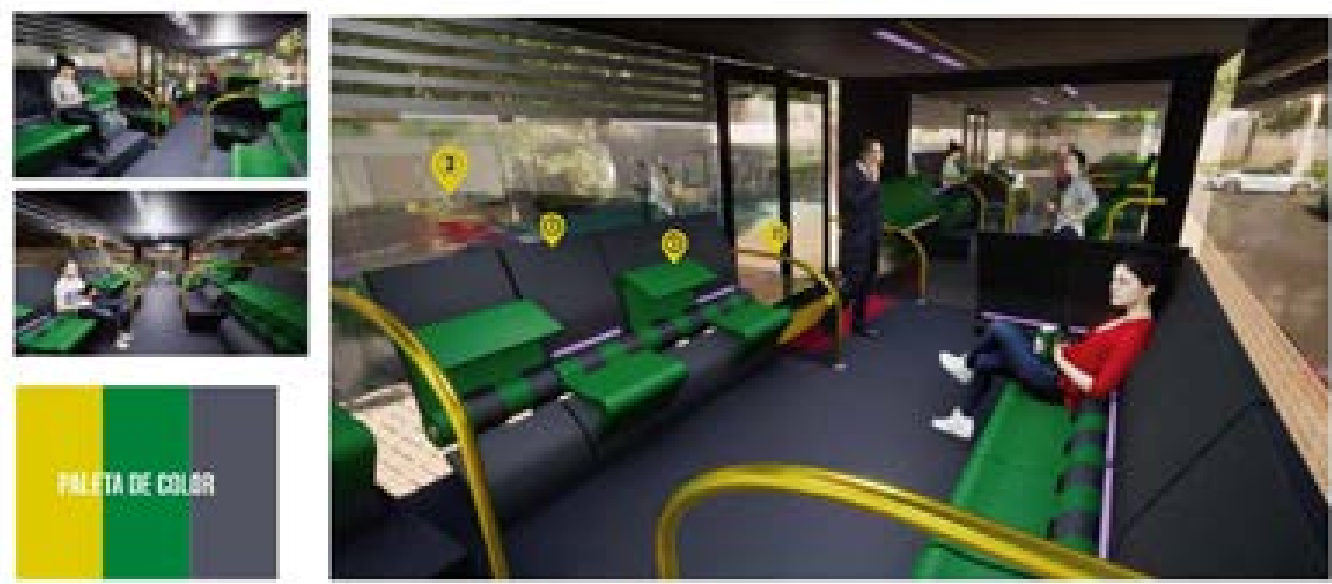

Fuente: Estudiantes Mariana Hernández y Daniela Cuervo, Taller 6, DI-UCP, 2020-02 

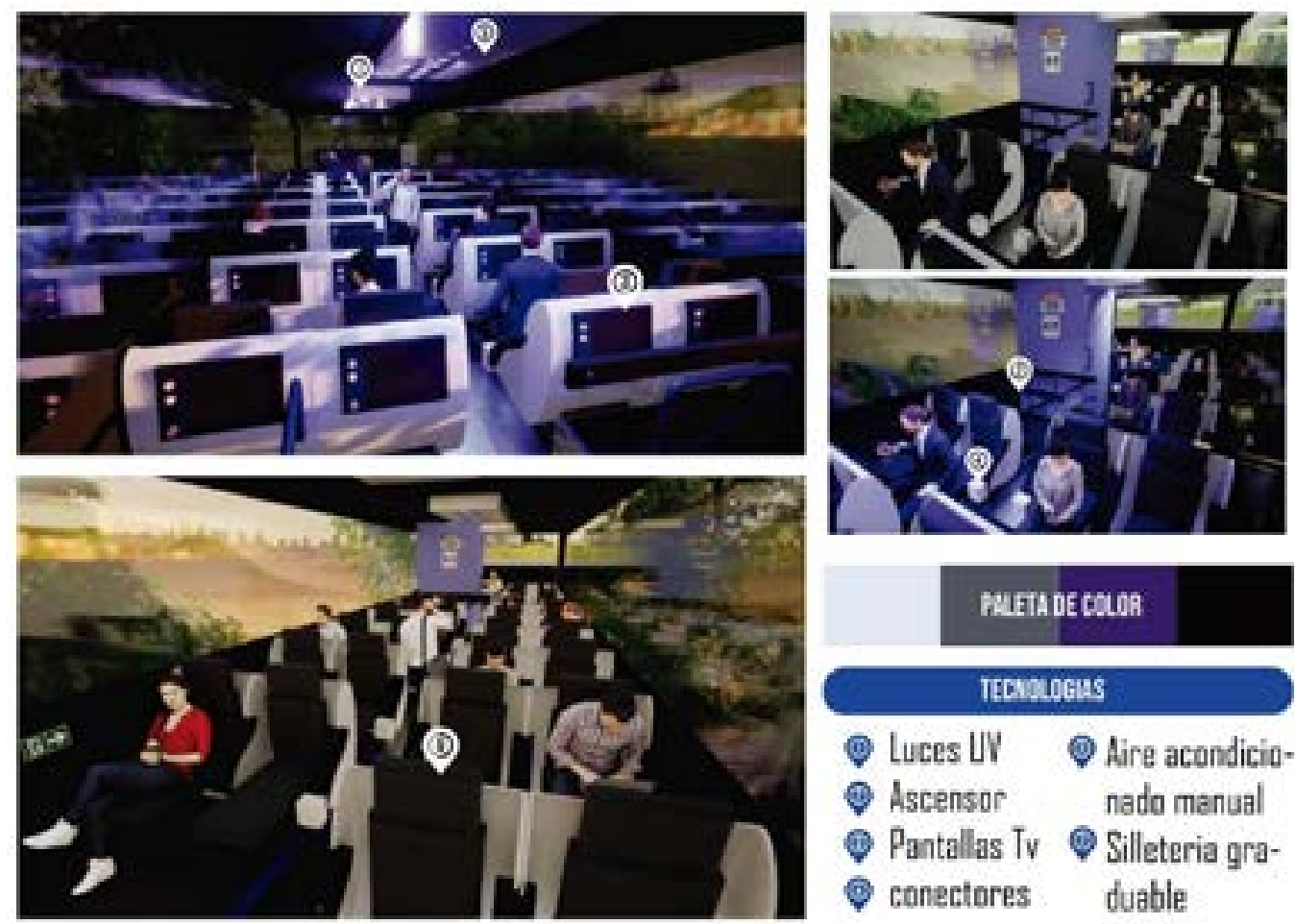

\section{FUETADE CAOQR}

\section{TERDOBgus}

(1) Luces UV

(1) Ascensor

(1) Pantallas Tv

다을 contores
(1) Aire acondicionado manual

- Silleteria graduable

Fuente: Estudiantes Mariana Hernández y Daniela Cuervo, Taller 6, DI-UCP, 2020-02

\section{Experiencia 2: HANKO Leather Crafts}

La segunda experiencia se llevó a cabo en la empresa HANKO Leather Crafts. Esta se dedica al diseño y fabricación de productos no convencionales en cuero, se encuentra ubicada en el municipio industrial de Dosquebradas, Risaralda. El proyecto se tituló Ciborg y tecnología vestible, su finalidad era desarrollar un objeto tecnológico vestible (accesorio no convencional en cuero) que le permitiera a una persona interrelacionarse como Cyborg con otros y con el contexto a partir de una adaptación o expansión tecnológica como intervención en producto y proceso para la empresa y su mercado regional. La iniciativa parte del concepto Diséñate a ti mismo, formulado por Moon Ribas y Neil Harbisson. 
Basado en dicho principio se propone una exploración trascendental y pionera desde la academia, en un área de reciente exploración en Colombia, con la meta de identificar nuevas oportunidades de diseño apoyados en las tecnologías que siguen avanzando velozmente y a las que los diseñadores industriales tendrán que adaptarse innovadoramente o caer en la obsolescencia de las industrias del pasado.

En el marco de la actual revolución 4.0 (Schwab, 2016), caracterizada por los avances en automatización de procesos industriales y la interconectividad de los artefactos a través del internet de las cosas o IoT-Internet of Things, surgen nuevas interrogantes sobre la participación del ser humano en las dinámicas productivas y de otro lado, en la interrelación persona-máquina en la cotidianidad. La simbiosis entre los humanos como organismos vivientes y los objetos como máquinas artificiales, se complementa cada vez más a través de la cibernética. Esto ocurre incluso en los niveles más simples de intervención tecnológica al aplicar dispositivos mecánicos y electrónicos a los objetos y accesorios que las personas portan habitualmente como exploración de nuevas percepciones sensoriales mediadas por la tecnología y que han conseguido materializar la unión entre lo cibernético y lo orgánico, como objetos diseñados para el futuro (Norman, 2010).

De este modo, se delimita una diferencia entre la tecnología que permite saber cosas y la tecnología que permite sentir cosas; en esa vía, la Fundación Cyborg se enfoca en los Sentidos Artificiales (SA), es decir, en el caso de esta última, la tecnología reúne los estímulos, pero la inteligencia es creada por el ser humano. En contraste, en el caso de la Inteligencia Artificial (IA) la inteligencia es creada por la máquina misma. Así en este escenario, se propone desarrollar productos innovadores para la empresa regional HANKO Leather Crafts, con la intención de agregar valor a las actividades cotidianas, los hobbies y la interrelación con otras personas en contexto de manera incremental o disruptiva. Esto debe propiciar interacciones mediadas por dispositivos 
tecnológicos de uso libre y bajo costo, que hagan parte de la simbiosis humanos-máquinas-entorno e incluso con otras especies que habitan inevitablemente en el Technium (Kelly, 2010) y que evolucionan hacia el transhumanismo.

De acuerdo con las ideas anteriores, los objetivos que se propusieron entre los docentes y el empresario para este proyecto fueron: En primer lugar, reconocer las tecnologías que puedan ser adaptadas de manera complementaria a objetos vestibles en una dimensión Cyborg; segundo, conceptualizar de manera reflexiva y crítica sobre las nuevas relaciones entre personas y tecnologías como posibilidades sensitivas, perceptivas y experienciales a través de la objetualidad y la interacción cibernética; tercero, diseñar un objeto tecnológico que favorezca la interacción de las personas con su entorno a través de nuevas sensibilidades, convirtiéndolo en un Cyborg; en cuanto al cuarto objetivo, se trata de desarrollar un producto real para una empresa que desarrolla accesorios no convencionales en cuero en la región; finalmente, se plantea que se debe proyectar cualitativamente un sistema productivo viable para este diseño, con una producción limitada de 100 unidades por mes.

En sintonía con dichos objetivos, los requerimientos preliminares del proyecto planteados colectivamente entre los docentes de las asignaturas de Taller y Automatización y el Gerente de HANKO se dividieron en 7 grandes aspectos. En primer lugar, se encuentra la investigación; en este aspecto se debe escribir un documento investigativo completo con todo el marco teórico del proyecto. Este debe evidenciar suficiencia en todos los términos, conceptos y tipologías relacionadas con el tema. El segundo aspecto es el de materiales y tecnología, el cual plantea que se debe utilizar cuero bovino, costuras en cáñamo a mano, dependiendo de las posibilidades tecnológicas, funcionales, interactivas y productivas de la empresa intervenida (corte láser, etc.). Se permite trabajar con otros 
materiales como: textiles, maderas, metales, polímeros, de manera complementaria y en un porcentaje máximo del 10\% en relación con el total en cuero. Electrónica básica (sensores y actuadores), entre otros que favorezcan la construcción del objeto tecnológico.

Con relación al tema de la calidad de fabricación, se debe tener en consideración un alto nivel de manufactura, acabados y funcionamiento real del objeto tecnológico. Con relación a la interacción cyborg se plantea que se debe pensar una interrelación creativa e innovadora del usuario como Cyborg en un contexto real, demostrable el día de sustentación en el escenario definido por el Programa de Diseño Industrial. Se tienen que demostrar tres simuladores (en fommy - cuerina, cuerotex, etc.) a escala real del objeto (1/1), previamente a la selección definitiva. El modelo seleccionado será desarrollado y construido para el día de la entrega final en escala real (1:1), debidamente ensamblado y funcional en cada una de sus partes. Por último, las consideraciones sobre el prototipo real, se debe elaborar con los materiales reales de producción para HANKO (cuero), elementos electrónicos, etc.

Después de varias asesorías remotas y una pre-entrega virtual, se presentaron algunos de los resultados más relevantes de esta experiencia académica con la empresa HANKO. Finalmente, se evaluaron los resultados de los productos diseñados de manera presencial y con los protocolos de bioseguridad en alternancia en las instalaciones de la UCP a finales de noviembre de 2020. Se evidencian los principales proyectos a continuación. 
Figura 7 Accesorio no convencional PRECISION para HANKO.

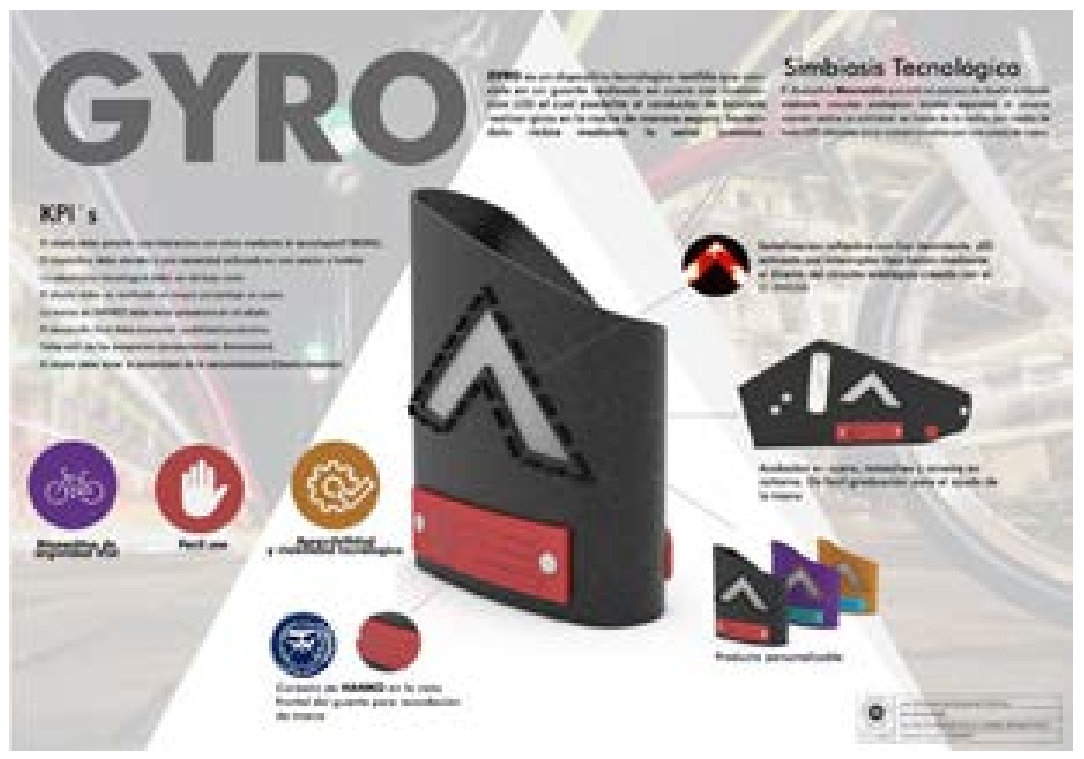

Fuente: Estudiante Daniela Cuervo, Taller 6, DI-UCP, 2020-02

Figura 9 Accesorio no convencional GYRO para HANKO.

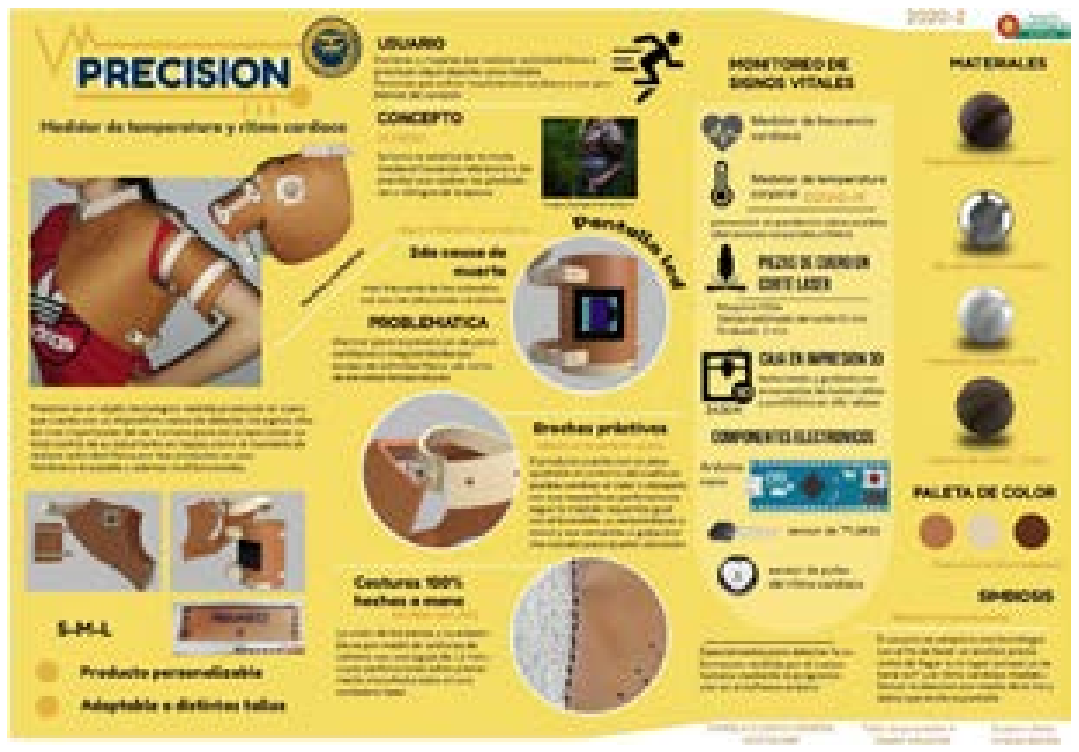

Fuente: Estudiante Karym Calle, Taller 6, DI-UCP, 2020-02 


\section{Reflexiones finales y conclusiones}

Uno de los mayores interrogantes enfrentados durante este distanciamiento de las aulas académicas ha sido cómo mantener la confianza y la credibilidad en las propuestas de diseño desde la virtualidad y la digitalidad para escenarios reales de la industria. Lo interesante de este reto es que las empresas que abrieron sus puertas siguen valorando los niveles de creatividad y la capacidad innovadora de los jóvenes diseñadores como fuente de respuestas a las cuestiones del diseño que enfrenta la industria regional. La posibilidad de mantener una relación con el sector productivo-industrial en la virtualidad al generar encuentros remotos para validar los resultados proyectuales desde lo académico, seguirá siendo una estrategia que fortalece la simbiosis universidad-empresa. Esto suma importancia a la interacción para consolidar aprendizajes significativos y la formación de diseñadores pertinentes para el ámbito productivo.

A pesar de las situaciones similares que viven la industria de manufactura y la educación al tener que enfrentar las condiciones de trabajo remoto y eventuales aislamientos alejados de los lugares tradicionales para la actividad presencial, aparecen modelos alternativos de encuentro e innovación basados en tecnologías que cada día son más asequibles y accesibles, y que favorecen el encuentro de diversos gremios de manera interdisciplinaria con resultados transdiciplinarios. La comunicación de los proyectos utilizando modelos digitales 2D y 3D a través del modelado con software y la renderización hiper-realista, favorecen la narrativa de los estudiantes a través de la virtualidad, al permitir una comunicación fluida en términos de representación visual y claridad conceptual para los empresarios.

Con relación al proyecto de Busscar, el escenario pandémico como oportunidad para diseñar soluciones para la movilidad y el transporte seguro de pasajeros plantea retos que requieren 
procedimientos de vigilancia tecnológica con mayor precisión debido

a la necesidad de adaptar tecnologías en el corto plazo para que los conceptos de diseño sugeridos puedan ser considerados por las empresas para su implementación. Por otra parte, la intervención en empresas de productos de menor escala y complejidad productiva requieren que las dinámicas de innovación en sus productos para la cotidianidad sean más ágiles y adaptables a los cambios en el mercado, lo cual puede ser beneficiado por las ventajas que ofrece la virtualidad y el encuentro remoto con equipos de co-creación junto a los empresarios interesados y procesos de co-diseño más robustos trabajando sincrónicamente en la distancia.

\section{Referencias bibliográficas}

Altshuller, G. (1997). Introducción a la innovación sistemática: TRIZ. Madrid: Internet Global.

Blu Radio. (01 de febrero de 2021). Educación virtual una necesidad impuesta que llega para quedarse. Bluradio. Recuperado de: https://www.bluradio.com/sociedad/educacion/educacionvirtual-una-necesidad-impuesta-que-llega-para-quedarse

Cardona Ossa, G. (2006). Metodologías y didácticas virtuales. Bogotá:

CINEV (Centro de Investigación en Educación Virtual).

Christensen, C. (2003). The innovator's solution: creating and sustaining successful growth. Boston, Massachusetts: Harvard Business School Publishing Corporation.

Hanington, B., \& Martin, B. (2019). Universal Methods of Design

(Expanded and Revised). Beverly, MA: Rockport Publishers.

Harvard Business Essentials. (2009). Innovator's Toolkit: 10 Practical Strategies to Help You Develop and Implement Innovation. Boston, Massachusetts: Harvard Business Press. 
Kelly, K. (2010). What technology wants. New York: Penguin Group.

Kumar, V. (2013). 101 Design Methods: A Structured Approach for Driving Innovation in Your Organization. Hoboken, New Jersey: Wiley.

Norman, D. (2010). El diseño de los objetos del futuro. La interacción entre el hombre y la máquina. Madrid: Paidós.

Schwab, K. (2016). La cuarta revolución industrial. Bogotá: Penguin Random House.

Valli, A. (2008). The design of natural interaction. Multimed Tools Appl, 38(3), 295-305. DOI: 10.1007/s11042-007-0190-z 